\title{
Luteolin, an aryl hydrocarbon receptor antagonist, alleviates diabetic retinopathy by regulating the NLRP/NOX4 signalling pathway: Experimental and molecular docking study
}

\author{
Y. YANG* ${ }^{*}$, M. ZHOU and H. LIU
}

Department of Ophthalmology, Affiliated Zhongshan Hospital of Dalian University, 116001, Dalian, Liaoning, China

\begin{abstract}
Objective: The present report evaluates the protective effects of luteolin against diabetic retinopathy (DR). Materials and methods: Diabetes was induced in rats by i.p. administration of $60 \mathrm{mg} / \mathrm{kg}$ of streptozotocin (STZ), followed by treatment with luteolin for 4 weeks. The effects of luteolin were determined based on the blood glucose and cytokine levels, and parameters of oxidative stress in retinal tissue of DR rats. The diameter of retinal vessels was estimated by fundus photography. A Western blot assay was used to determine the expression of apoptotic proteins and Nod-like receptor 3 (NLRP3) pathway proteins in the retina of DR rats. A molecular docking study was performed to evaluate the interaction between luteolin and NLRP3. Results: The level of blood glucose was reduced in the luteolin-treated group compared with the DR group. Reductions in cytokines and oxidative stress were observed in the retinal tissues of the luteolin-treated group relative to the DR group. Moreover, treatment with luteolin reduced the expression of NLRP1, NOX4, TXNIP, and NLRP3 proteins, and ameliorated the altered expression of apoptotic proteins in the retina of DR rats. Conclusion: In conclusion, luteolin prevents retinal apoptosis in DR rats by regulating the NLRP/NOX4 signalling pathway.
\end{abstract}

\section{KEYWORDS}

luteolin, diabetic retinopathy, caspase-1, streptozotocin, oxidative stress

\footnotetext{
* Corresponding author. Department of ophthalmology, Affiliated Zhongshan Hospital of Dalian University, No. 6 Jiefang Street, 116001, Dalian, Liaoning China. Tel./fax: +86 0411 62893555. E-mail: yang_7805@yahoo.com
} 


\section{INTRODUCTION}

Diabetes is a chronic metabolic disorder characterized by an increased glucose level that affects approximately 170 million people worldwide [1]. Left uncontrolled for a long time, hyperglycaemia leads to several chronic complications including diabetic retinopathy (DR) [2]. DR is a form of progressive retinal microvasculature damage and a major cause of loss of vision worldwide [3]. Retinal damage in DR is classified into two primary types: proliferative and nonproliferative [4]. Diabetic macular oedema occurs in retinal tissues in non-proliferative DR. However, alterations in the growth and formation of blood vessels also occur under low-oxygen conditions in the proliferative type [5]. Changes in vascular permeability cause injury to the macula and an accumulation of fluids in retinal tissues, which are characteristic of DR [6].

Several pathways are involved in DR, as reactive oxygen species (ROS) formation is enhanced due to alterations in the citric acid and glycolysis cycles as a result of hyperglycaemia. The activities of ROS-generating enzymes, such as NADPH oxidase 4 (NOX4) are enhanced by inflammatory processes promoted by an increase in cytokine level [7]. The literature suggests that the inflammation involved in the development of DR, and retinal tissue necrosis and apoptosis is caused by elevated caspase-1 expression [8]. Nod-like receptors (NLRP)-1 and -3 promote the expression of IL-18 and IL-1 $\beta$, which activate caspase-1 [9]. Collectively, this contributes to progressive damage to the retinal tissue in DR. Current therapies available for DR have several limitations. Thus, there is a clear need for the development of new drug targets and therapies for DR.

Several molecules of natural origin have shown potent effects against chronic disorders, including diabetic complications. Luteolin is a flavonoid that acts as an antagonist of the aryl hydrocarbon receptor isolated from Eclipta alba [10]. Previous reports have described genetically modified rats in which aryl hydrocarbon receptor deficiency enhanced insulin sensitivity, and its antagonists protected against diabetes [11]. Luteolin reportedly has antidiabetic, antiinflammatory, anti-obesity, antioxidant, and neuroprotective effects [12, 13]. Moreover, luteolin prevents vascular injury and protects against atherosclerosis by regulating the NLRP-3 pathway [14]. The anti-inflammatory properties of luteolin are reflected in its ability to reduce levels of IL-6 and NF-kBp65, and thereby exert anticancer effects [15]. The present report evaluates the protective effect of luteolin against retinal injury in DR.

\section{MATERIAL AND METHODS}

\section{Animals}

Healthy (200-250-g) Wistar rats were maintained under controlled conditions as per guidelines of Association for the Assessment and Accreditation of Laboratory Animal Care International (AAALAC) for experimentation and animal use [16] (12-h light/dark cycle, $25 \pm 3{ }^{\circ} \mathrm{C}$, and $60 \pm$ $5 \%$ humidity). All experimental protocols reported here were approved by the Institutional Animal Ethics Committee of Affiliated Zhongshan Hospital of Dalian University, China.

\section{Experimental}

Animals of different groups were fasted overnight before the i.p. administration of $60 \mathrm{mg} / \mathrm{kg}$ of streptozotocin (STZ) for the induction of diabetes. Diabetes was confirmed based on the serum 
level of glucose, determined using an autoanalyzer (Biobase Biodustry (Shandong) Co., Ltd., Shandong, China). The rats were randomly divided into three groups: the normal group, DR group, and luteolin group. The luteolin group received luteolin $50 \mathrm{mg} / \mathrm{kg}$ p.o. for 4 weeks after the induction of DR. At the end of the treatment period, the blood glucose level was determined in all groups.

Fundus photography. Isoflurane was used to anesthetize the rats, and tropicamide was applied to the eyes to dilate the pupils. The rats were placed beneath a retinal camera, and a clear image of the retina was obtained. Image-Pro Plus software (Media Cybernetics, Silver Spring, MD, USA) was used to determine the diameter of the blood vessels.

Measurement of oxidative stress. A riboflavin-sensitized method was used to assess the activity of superoxide dismutase (SOD): $2 \mathrm{~mL}$ of reaction mixture containing $6.6 \times 10^{-3} \mathrm{M}$ EDTA, $10^{-2} \mathrm{M}$ methionine, $1.76 \times 10^{-4} \mathrm{M}$ nitroblue tetrazolium, $2 \times 10^{-5} \mathrm{M}$ sodium cyanide $(\mathrm{NaCN})$ and $2 \times 10^{-6} \mathrm{M}$ riboflavin in $50 \mathrm{mM}$ phosphate buffer ( $\mathrm{pH} 7.8$ ) was added to each tube, followed by $5 \mu \mathrm{L}$ sample. Tubes were placed for a period of $10 \mathrm{~min}$ under a $15 \mathrm{~W}$ lamp and the absorbance was measured at $460 \mathrm{~nm}$. Data of activity of SOD were expressed as U/mg protein [17].

The method reported by Ohkawa was used to determine the level of lipid peroxidation in retinal tissues. Tissue homogenate $(0.5 \mathrm{~mL})$ was added to $1 \mathrm{~mL}$ of $0.67 \%$ thiobarbituric acid (TBA) and $5 \mathrm{~mL}$ of $20 \%$ trichloroacetic acid (TCA) in aqueous solution. The mixture was heated in a boiling water bath for $15 \mathrm{~min}$ and then cooled. The mixture was further treated with $4 \mathrm{~mL}$ of $n$-butanol and then centrifuged for $15 \mathrm{~min}$ at 3,000 rpm. The malondialdehyde (MDA) level was determined in retinal tissue at a wavelength of $530 \mathrm{~nm}$ in a spectrophotometer (Shimadzu corporation, Japan) [18].

Measurement of cytokines. The levels of cytokines, namely NF- $\kappa \mathrm{B}$, IL- $1 \beta$, IL-6, and TNF- $\alpha$ in the eye tissue were determined using an ELISA kit (ThermoFisher Scientific, USA) according to the manufacturer's recommendations and evaluated by an ELISA reader (BioTek, Winooski, Vermont, USA).

Western blotting. The expression levels of caspase-1, Bax, Bcl2, IL-1 $\beta$, NLRP1, NOX4, TXNIP, and NLRP3 were measured in isolated eye tissues using Western blotting. RIPA buffer containing Phosphatase Inhibitor Cocktail and Halt Protease was used to homogenize the isolated retinal tissue. The protein content in tissue homogenates was determined using the BCA assay kit, and proteins were separated by $10 \%$ sodium dodecyl sulphate-polyacrylamide gel electrophoresis. The separated proteins were transferred to nitrocellulose membrane, which was then blocked in $5 \%$ blocking reagent. The membrane was incubated with primary antibodies targeting caspase-1, Bax, Bcl2, IL-1 $\beta$, NLRP1, NOX4, TXNIP, and NLRP3 (ThermoFisher Scientific, USA) overnight at $4{ }^{\circ} \mathrm{C}$. After washing with PBS, the membrane was incubated with goat secondary antibodies conjugated to horseradish peroxidase, and protein expression was detected using a chemiluminescence kit. Chemiluminescence was detected with an Enhanced Chemiluminescence (ECL) Western blot detection kit (Amersham Pharmacia Biotech, Buckinghamshire, UK). To confirm that equal amounts of protein were electrophoresed and transferred to the membrane, staining with Coomassie Brilliant Blue (CBB) R-250 was performed. 
Estimation of caspase-1 activity. A caspase-1 colorimetric assay kit was used to determine the caspase- 1 activity in the retinal tissue, as per the manufacturer's instructions (SigmaAldrich Ltd., USA). Data are presented as caspase-1 activity per unit weight of protein.

\section{Histopathology study}

Harvested rat eye tissues were fixed by seeding in $4 \%$ paraformaldehyde, and 4 - $\mu \mathrm{m}$-thick retinal tissues were sectioned. Tissue sections were rehydrated with ethanol, and haematoxylin-eosin staining was used to stain each section. Light microscopy was applied to observe pathological changes in the eye tissue.

Homology model of NLRP3. The protein sequence of the NLRP3 protein was obtained from the NCBI database for preparation of the homology model. The SWISS-MODEL server was used to develop the homology model for the NLRP3 protein by SWISS modeling. Several software packages were used to perform the investigation, including ACD ChemSketch (ACD Labs, Ontario, Canada)), the PyMol Molecular Graphics System (version 2.4.0; Schrodinger, LLC, New York, NY, USA), and Autodock Vina (ver. 1.5.6; (The Scripps Research Institute, La Jolla, CA, USA). A universal protein source was used to sequence the NLRP3 protein. Target sequences against the primary amino acid sequence were identified via BLAST search. The bestquality templates were selected for building the homology model. Autodock Vina was used to prepare the ligand for the docking study.

Preparation of proteins and ligands, and molecular docking. The two-dimensional ligand structure was obtained from the PubChem database and converted into a pdb file using Open Babel software (http://openbabel.org/wiki/Main_Page). Autodock Vina was used to prepare ligands for the docking study by removing water molecules and adding hydrogen, and then modulating the charges using the Kollman approach; finally, the pdbqt file was obtained. The molecular docking simulation of luteolin was done using Autodock based on the Kollman and Gasteiger charges in the ligand and protein. The grid map was created using AutoGrid 4 in AutoDock Vina. After preparing the grid box, the area of the protein structure to be mapped was defined. The grid box dimensions ( $x, y$, and $z$ coordinates) for NLRP3 were 79.658853, 99.032284, and 93.160451, respectively. A Lamarckian genetic algorithm was used for minimization and optimization of energies in the docking simulation process.

\section{Statistical analysis}

All data are expressed as mean \pm standard error of the mean (SEM; $n=10$ ), and statistical analysis was performed using one-way analysis of variance (ANOVA). Post hoc comparisons of means were carried out with Dunnett's test using GraphPad Prism software (ver. 6.1; GraphPad Software, Inc., San Diego, CA, USA). Data with $P$ value $<0.05$ were considered statistically significant.

\section{RESULTS}

\section{Luteolin reduces the blood glucose level}

The serum glucose level of luteolin-treated DR rats was estimated on days 3 and 8, as shown in Fig. 1. An increase in the serum glucose level of the DR and luteolin groups compared with the 


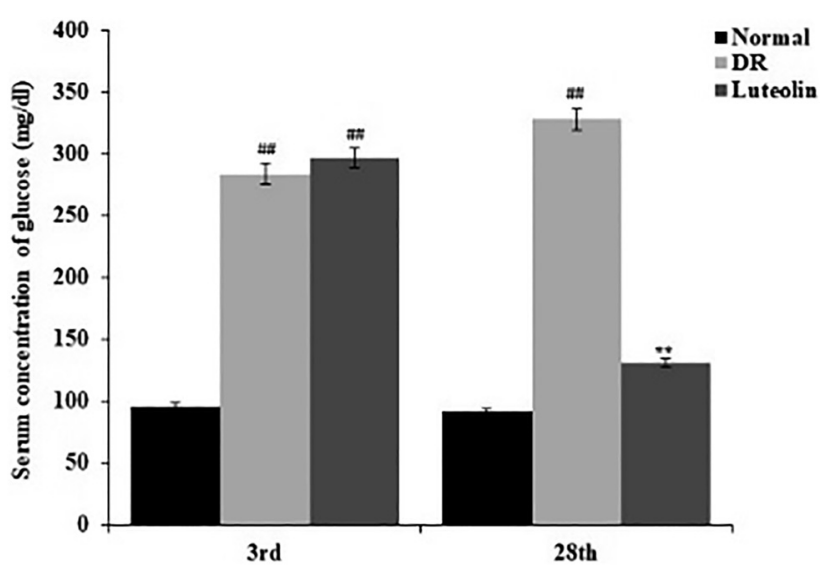

Fig. 1. Luteolin treatment reduces the serum glucose level in DR rats.

Mean \pm SEM $(n=10) ;{ }^{\# \#} P<0.01$ compared with the normal group; ${ }^{* *} P<0.01$ compared with the DR group

normal group was observed after 3 days of STZ administration. The serum glucose level was further enhanced in the DR group compared with the normal group. However, treatment with luteolin reduced the concentration of glucose in the serum of DR rats.

\section{Luteolin reduces the diameter of retinal vessels}

The effect of luteolin on the diameter of retinal vessels in the DR rats is shown in Fig. 2. On fundus photography, the retinal arteriolar diameter was $74.9 \mu \mathrm{m}$ in the DR group and $38.6 \mu \mathrm{m}$ in the normal group. In the luteolin-treated group, the retinal arteriolar diameter was decreased to $46.1 \mu \mathrm{m}$ compared with the DR group.

\section{Luteolin reduces oxidative stress}

Parameters of oxidative stress, such as MDA level and SOD activity were estimated in the retinal tissue of luteolin-treated DR rats. The MDA level in wet tissue was $10.4 \mu \mathrm{M} / \mathrm{mg}$ higher, whereas the SOD level in wet tissue was $0.73 \mathrm{U} / \mathrm{mg}$ lower in the tissue homogenate of the DR group compared with the normal group. However, treatment with luteolin reduced the magnitude of the change in MDA level and SOD activity in the retinal homogenate of DR rats (Fig. 3).

\section{Luteolin reduces the level of cytokines}

Cytokine levels in the retinal tissues of luteolin-treated DR rats were determined, as shown in Fig. $4 \mathrm{~A}$ and B. ELISA was used to estimate the IL- $1 \beta$, IL- 6 , TNF- $\alpha$, and NF- $\kappa$ B levels in the retinal tissues of luteolin-treated DR rats. There was an increase in cytokine concentrations in the tissue homogenate of the DR group relative to the normal group. The cytokine concentrations were reduced in the luteolin-treated group compared with the DR group (Fig. 4A). IL$1 \beta$ expression in the tissue homogenate of the DR group was enhanced compared with that in the normal group. Finally, the expression of IL-1 $\beta$ in the retinal tissues of the luteolin-treated group was lower than that in the DR group (Fig. 4B). 


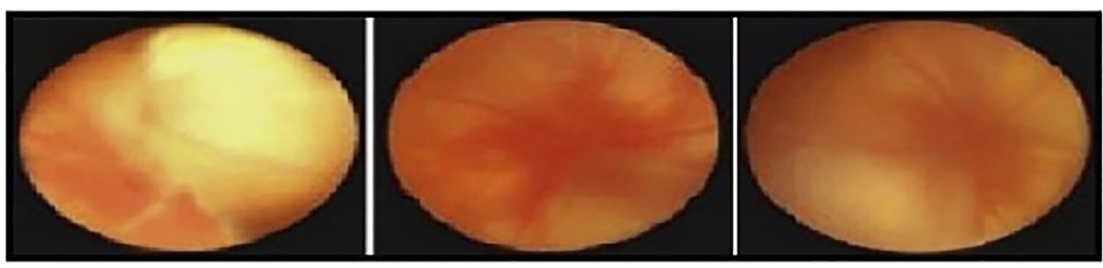

Normal

DR

Luteolin

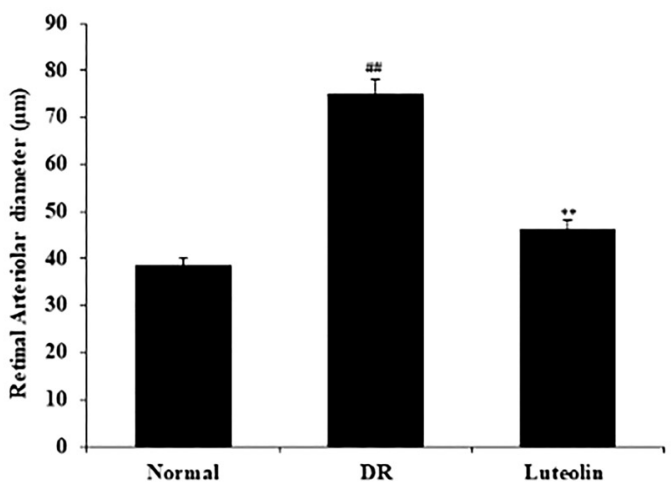

Fig. 2. Effect of luteolin on fundus photography and the diameter of retinal vessels in DR rats. Mean \pm SEM $(n=10) ;{ }^{\# \#} P<0.01$ compared with the normal group; ${ }^{* *} P<0.01$ compared with the DR group
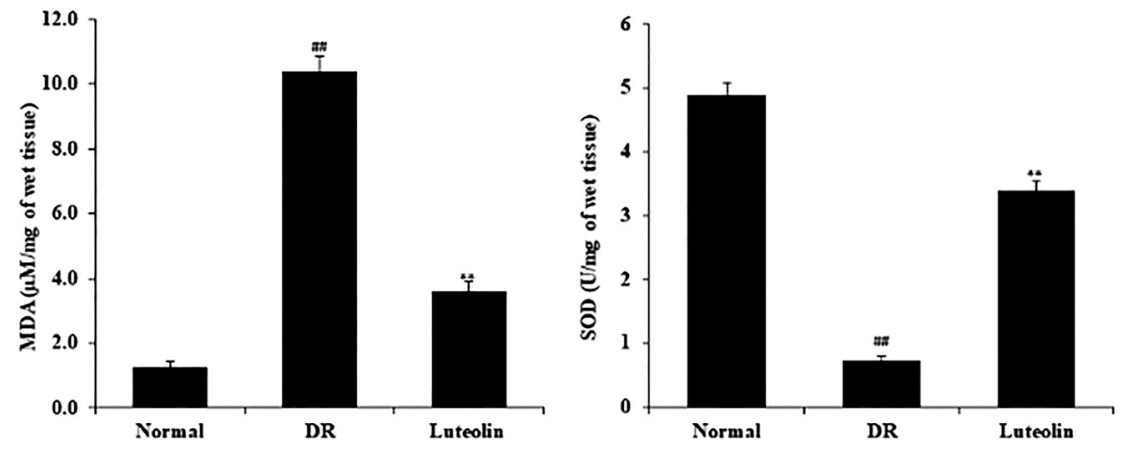

Fig. 3. Luteolin reduces oxidative stress in retinal tissues in DR rats.

Mean \pm SEM $(n=10) ;{ }^{\# \#} P<0.01$ compared with the normal group; ${ }^{* *} P<0.01$ compared with the DR group

\section{Luteolin reduces retinal apoptosis}

Retinal cell apoptosis was determined by estimating the expression levels of the caspase-1, Bax, and Bcl-2 proteins, and the activity of caspase- 1 in the retina of luteolin-treated DR rats (Fig. 5A and B). Caspase-1, Bax, and Bcl-2 protein expression levels were estimated using a Western blot assay, as shown in Fig. 5A. The expression levels of caspase-1 and Bax were increased, whereas 
A

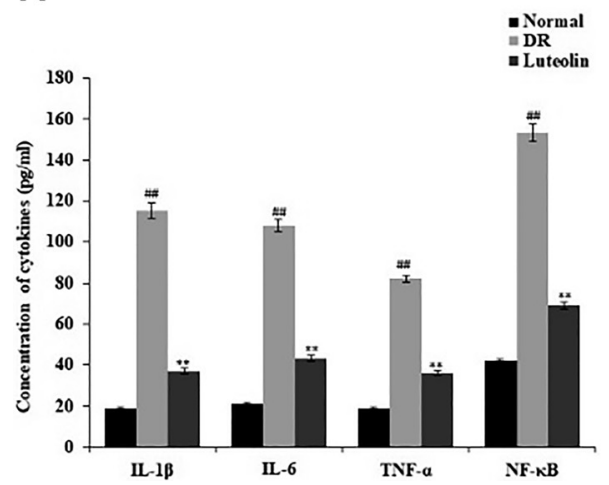

B
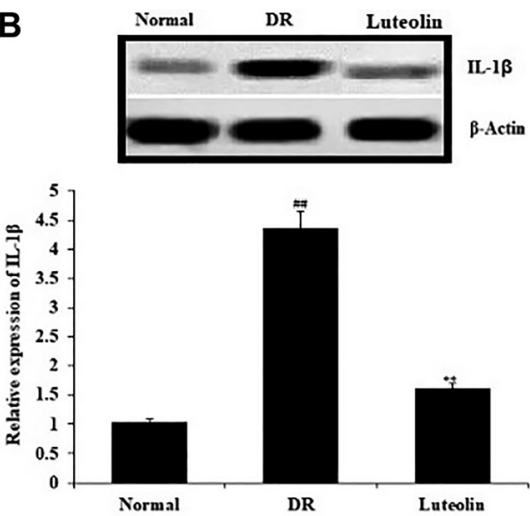

Fig. 4. Luteolin reduces inflammatory cytokines in retinal tissues in DR rats. A: Concentrations of the cytokines IL- $1 \beta$, IL- 6 , TNF- $\alpha$, and NF- $\kappa$ B in the retinal tissues, as revealed by ELISA; B: Relative expression of IL- $1 \beta$, as revealed by Western blot assay.

Mean \pm SEM $(n=10) ;{ }^{\# \#} P<0.01$ compared with the normal group; ${ }^{* *} P<0.01$ compared with the DR group

that of $\mathrm{Bcl}-2$ protein was decreased in the tissue homogenate of the DR group relative to the normal group. However, treatment with luteolin attenuated the altered expression of Bax, caspase-1, and Bcl-2 proteins in DR rats. The activity of the caspase-1 enzyme was also estimated in the tissue homogenate of DR rats, as shown in Fig. 5B. The activity of caspase-1 was enhanced in the retina in the DR group compared with the normal group. The activity of caspase-1 enzyme in the retina in the luteolin-treated group was reduced compared with the DR group.

\section{Luteolin facilitates NLRP1/NOX4 pathway activation}

Luteolin affected the expression of the NLRP1, NOX4, TXNIP, and NLRP3 proteins in the retinal tissue of DR rats. The expression of the NLRP1, NOX4, TXNIP, and NLRP3 proteins in the retinal tissue homogenate of the DR group was significantly enhanced compared with the normal group. Expression levels of the NLRP1, NOX4, TXNIP, and NLRP3 proteins were reduced significantly $(P<0.01)$ in the retinas of luteolin-treated rats compared with DR rats (Fig. 6).

\section{Luteolin ameliorates pathological changes in retinal tissue}

Histopathological changes in the retina in luteolin-treated DR rats were observed by $\mathrm{H} \& \mathrm{E}$ staining. In the DR group, histopathology of the retina suggested oedema, congestion, and vascular structure enhancement, as well as a loss of ganglion cells. However, treatment with luteolin reversed the histopathological changes in the retina in DR rats (Fig. 7).

\section{Effect of luteolin on the NLRP3 protein}

Luteolin had high binding affinity with the NLRP3 protein. An in vivo and in vitro molecular docking study was performed using BLAST and homology modeling, followed by ligand and 

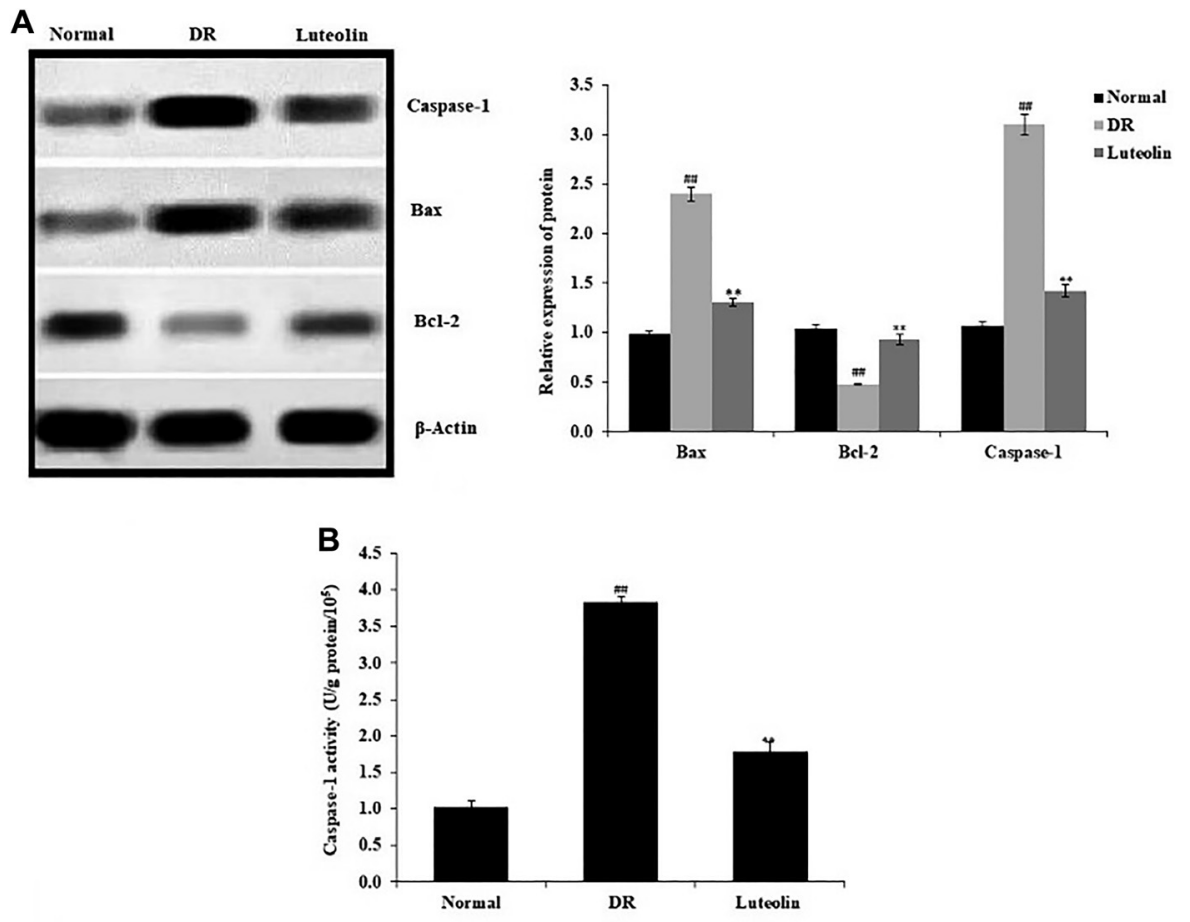

Fig. 5. Luteolin reduces apoptosis in the retinal tissues of DR rats. A: Relative expression of Bax, Bcl-2, and Caspase-1 in retinal tissues, as revealed by Western blot assay; B: Activity of caspase-1, as revealed by caspase-1 assay.

Mean \pm SEM $(n=10) ;{ }^{\# \#} P<0.01$ compared with the normal group; ${ }^{* *} P<0.01$ compared with the DR group
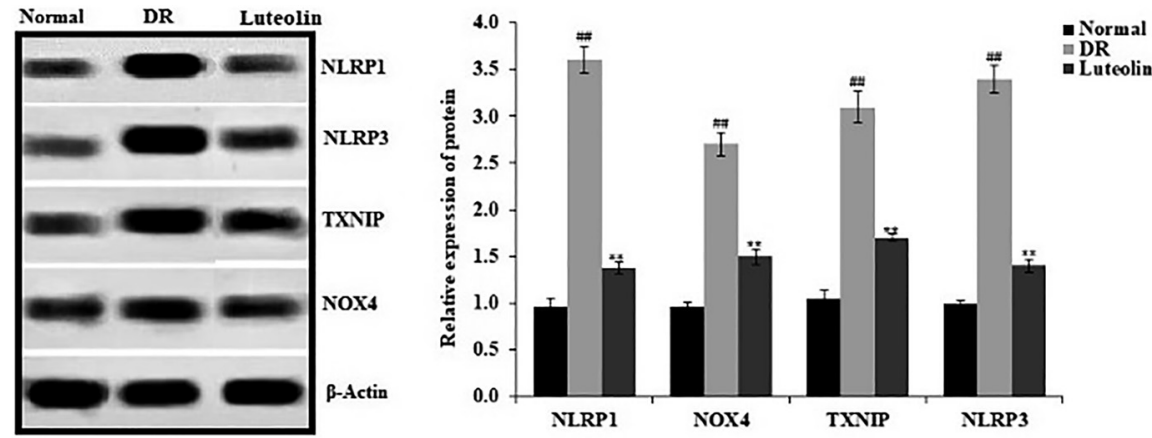

Fig. 6. Luteolin reduces the expression of NLRP1, NOX4, TXNIP, and NLRP3 in retinal tissues in DR rats. Mean \pm SEM $(n=10) ;{ }^{\# \#} P<0.01$ compared with the normal group; ${ }^{* *} P<0.01$ compared with the DR group 


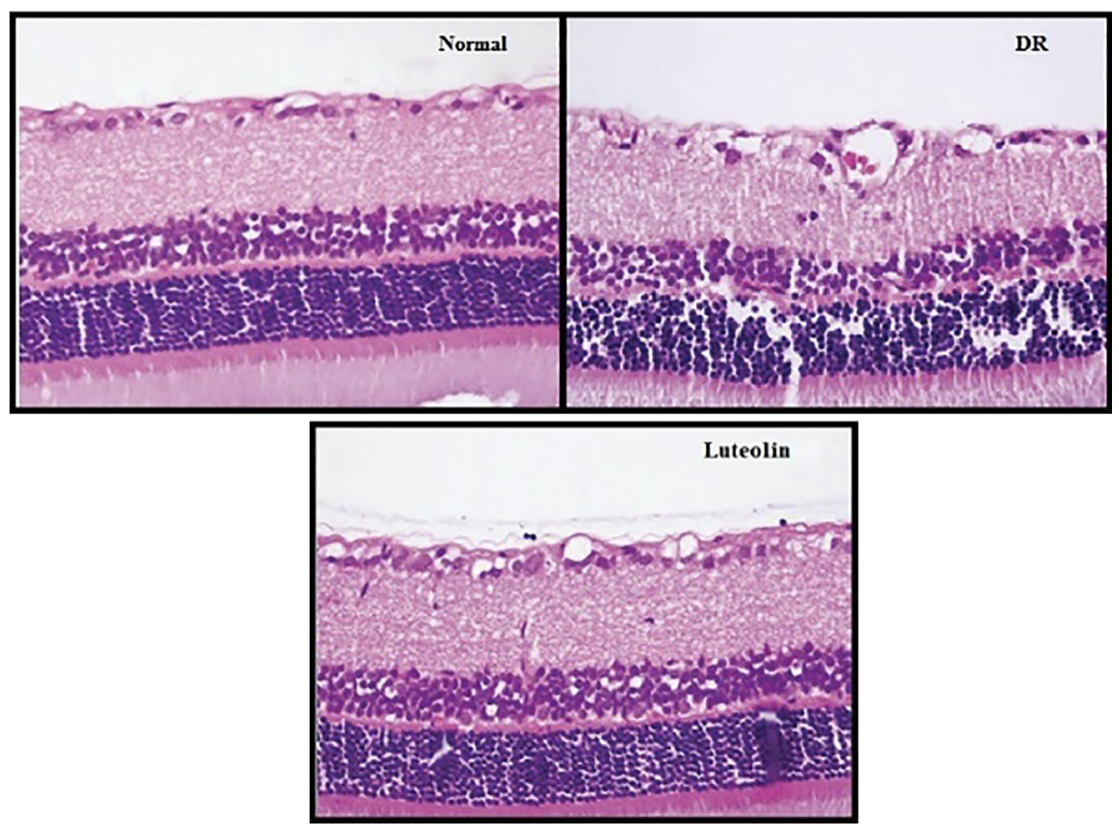

Fig. 7. Luteolin reduces histopathological changes in retinal tissues in DR rats

protein preparation. A luteolin molecular docking simulation was done using Autodock based on the Kollman and Gasteiger charges in both the ligand and the protein. The results showed higher binding affinity for luteolin with both in vivo-confirmed proteins and the NLRP3 protein. The binding energy data showed that luteolin was bound to NLRP3 (Table 1). Three-dimensional and two-dimensional structural analysis of the protein revealed a solid area, representing the binding area between the ligand and the NLRP3 protein (Fig. 8A and B).

Table 1. Docking scores for NLRP3 protein with ligand molecule Luteolin

\begin{tabular}{lccc}
\hline Mode of ligand & Affinity $(\mathrm{kcal} / \mathrm{mol})$ & Distance from rmsd l.b & Best mode rmsd u.b \\
\hline 1 & -8.2 & 0.000 & 0.000 \\
2 & -8.1 & 3.403 & 5.091 \\
3 & -7.9 & 3.350 & 7.416 \\
4 & -7.8 & 3.484 & 5.896 \\
5 & -7.7 & 1.169 & 2.689 \\
6 & -7.6 & 1.737 & 6.978 \\
7 & -7.4 & 19.137 & 19.502 \\
8 & -7.1 & 1.458 & 6.771 \\
9 & -7.0 & 3.122 & 4.491 \\
\hline
\end{tabular}

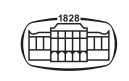



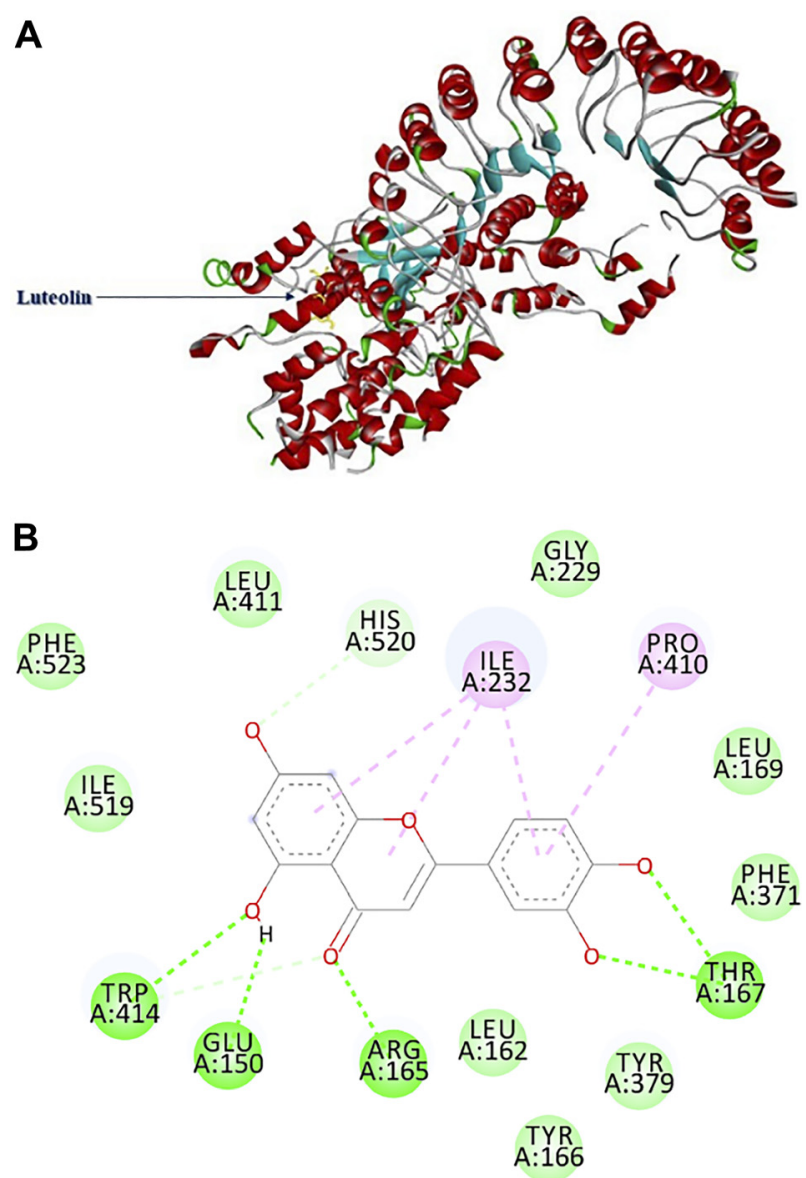

Fig. 8. In silico molecular docking showing the interaction of NLRP3 protein with luteolin. The solid area in the protein structures represents the area of interaction with the ligand. A: Three-dimensional molecular structure; B: Two-dimensional molecular structure

\section{DISCUSSION}

Diabetic retinopathy is a major complication of diabetes, contributing to the loss of vision. Management of diabetic retinopathy is still a challenge, and novel therapies are needed. Therefore, the present report evaluated the protective effect of luteolin against diabetic retinopathy (specifically, its effect on blood glucose and cytokine levels), as well as parameters of oxidative stress in the retinal tissue of diabetic retinopathy rats.

Alterations in the retinal microvascular circulation lead to changes in the retinal structure in diabetes mellitus [19]. In diabetic retinopathy, changes in vascularization, oedema, hyperproliferation of endothelial cells, and increased vascular permeability result in the loss of vision. The literature indicates that luteolin regulates blood glucose in diabetic rats [11], and the data of 
the present study also suggest that treatment with luteolin reduces the serum glucose concentration in diabetic retinopathy rats.

Diabetic retinopathy changes the flow of blood to the retina, resulting in an increase in ROS production, which in turn enhances oxidative stress [20]. Oxidative stress and ROS production are increased by overexpression of NOX4, and in a previous report the expression of NOX4 was found to be enhanced in diabetic retinopathy [21]. The results of the present study support this finding. Moreover, hyperglycaemia enhances the expression of the thioredoxin-interacting protein (TxNIP), and a recent study reported that $\beta$-cell failure was not seen in TxNIP-deficient mice [22]. This finding suggested that expression of the TXNIP protein was enhanced in the diabetic retinopathy group compared with the normal group. The expression of TXNIP and NOX4 was reduced, as was oxidative stress, in the retinas of the luteolin-treated group compared with the diabetic retinopathy rats.

Oxidative stress increases due to changes in the expression of cytokines, such as IL-1 $\beta$ and IL-18, which are activated by higher levels of NLRP-1 and -3 [23]. NLRP-1 and -3 enhance the activity of the caspase- 1 enzyme and alter apoptotic protein expression patterns. Previous studies indicated that activation of NLRP-1 or -3 was involved in the pathogenesis of diabetic retinopathy [24]; the data of the present study support this conclusion. Our results indicate that treatment with luteolin reduces the increase in cytokine levels and the expression of NLRP-1 and -3 in the retinal tissue of diabetic retinopathy rats. Alterations in the expression of NLRP 1 and interleukins activate apoptosis in the retina [25]. Moreover, our data show that retinal apoptosis contributes to the loss of vision, such that diabetic retinopathy is a major cause of blindness. The expression of apoptotic proteins and the activity of caspase- 1 showed greater reductions in the luteolin-treated group than in the diabetic retinopathy group. Moreover, the histopathological study revealed that treatment with luteolin attenuates the pathological changes that occur in the retina in diabetic retinopathy rats. Our report presented here has a few limitations, such as the study didn't evaluate the effect of luteolin clinically. Moreover, the dosage form for the administration of luteolin also needs to be developed, and the pharmacokinetic data of luteolin need to be studied.

\section{CONCLUSION}

In conclusion, a diabetic retinopathy rat model has been successfully established in this report, which will contribute to the therapeutic development of drugs for diabetic retinopathy. The present report also establishes the evidence of contribution of the NLRP/NOX pathway in the development of retinal injury in diabetic retinopathy, and demonstrates that treatment with luteolin ameliorates retinal apoptosis in diabetic retinopathy rats by regulating the NLRP1/ NLRP3/ NOX4 signalling pathway. Thus, luteolin could be used clinically for the management of diabetic retinopathy.

Ethics approval and consent to participate: All experimental protocols performed in this study were approved by the Institutional Animal Ethics Committee of the Affiliated Zhongshan Hospital of Dalian University, China (IAEC/ZH-DU/2019/07).

Competing interests: The authors have no competing interests to declare. 
Availability of data and materials: All data generated and analysed during this study are included in this article.

\section{ACKNOWLEDGEMENT}

The authors are thankful to the Affiliated Zhongshan Hospital of Dalian University, China, for providing the facilities necessary to conduct the present study.

\section{REFERENCES}

1. Dorcely B, Katz K, Jagannathan R, Chiang SS, Oluwadare B, Goldberg IJ, et al. Novel biomarkers for prediabetes, diabetes, and associated complications. Diabetes Metab Syndr Obes 2017; 10: 345-61.

2. Chawla A, Chawla R, Jaggi S. Microvascular and macrovascular complications in diabetes mellitus: distinct or continuum? Indian J Endocrinol Metab 2016; 20(4): 546-51.

3. Duh EJ, Sun JK, Stitt AW. Diabetic retinopathy: current understanding, mechanisms, and treatment strategies. JCI Insight 2017; 2(14): e93751.

4. Sharma Y, Saxena S, Mishra A, Saxena A, Natu SM. Nutrition for diabetic retinopathy: plummeting the inevitable threat of diabetic vision loss. Eur J Nutr 2017; 56: 2013-27.

5. Shin ES, Sorenson CM, Sheibani N. Diabetes and retinal vascular dysfunction. J Ophthalmic Vis Res 2014; 9(3): 362-73.

6. Kusuhara S, Fukushima Y, Ogura S, Inoue N, Uemura A. Pathophysiology of diabetic retinopathy: the old and the new. Diabetes Metab J 2018; 42(5): 364-76.

7. Mittal M, Siddiqui MR, Tran K, Reddy SP, Malik AB. Reactive oxygen species in inflammation and tissue injury. Antioxid Redox Signal 2014; 20(7): 1126-67.

8. Rübsam A, Parikh S, Fort PE. Role of inflammation in diabetic retinopathy. Int J Mol Sci 2018; 19(4): 942.

9. Lim RR, Wieser ME, Ganga RR, Barathi VA, Lakshminarayanan R, Mohan RR, et al. NOD-like receptors in the eye: uncovering its role in diabetic retinopathy. Int J Mol Sci 2020; 21(3): 899.

10. Zhang S, Qin C, Safe SH. Flavonoids as aryl hydrocarbon receptor agonists/antagonists: effects of structure and cell context. Environ Health Perspect 2003; 111(16): 1877-82.

11. Wang C, Xu CX, Krager SL, Bottum KM, Liao DF, Tischkau SA. Aryl hydrocarbon receptor deficiency enhances insulin sensitivity and reduces PPAR- $\alpha$ pathway activity in mice. Environ Health Perspect 2011; 119(12): 1739-44.

12. Choi JS, Islam MN, Ali MY, Kim YM, Park HJ, Sohn HS, et al. The effects of C-glycosylation of luteolin on its antioxidant, anti-Alzheimer's disease, anti-diabetic, and anti-inflammatory activities. Arch Pharm Res 2014; 37(10): 1354-63.

13. Kwon EY, Choi MS. Luteolin targets the toll-like receptor signaling pathway in prevention of hepatic and adipocyte fibrosis and insulin resistance in diet-induced obese mice. Nutrients 2018; 10(10): 1415.

14. Zhang BC, Li Z, Xu W, Xiang CH, Ma YF. Luteolin alleviates NLRP3 inflammasome activation and directs macrophage polarization in lipopolysaccharide-stimulated RAW264.7 cells. Am J Transl Res 2018; 10(1): 265-73.

15. Lin Y, Shi R, Wang X, Shen HM. Luteolin, a flavonoid with potential for cancer prevention and therapy. Curr Cancer Drug Targets 2008; 8(7): 634-46. 
16. Guide for the care and use of laboratory animals: eighth edition committee for the update of the guide for the Care and use of laboratory animals. National Research Council; 2010; ISBN: 0-309-15401-4.

17. Janknegt PJ, Rijstenbil JW, van de Poll WH, Gechev TS, Buma AG. A comparison of quantitative and qualitative superoxide dismutase assays for application to low temperature microalgae. J Photochem Photobiol B 2007; 87(3): 218-26.

18. Ohkawa H, Ohishi N, Yagi K. Assay for lipid peroxides in animal tissues by thiobarbituric acid reaction. Anal Biochem 1979; 95(2): 351-8.

19. Homme RP, Singh M, Majumder A, George AK, Nair K, Sandhu HS, et al. Remodeling of retinal architecture in diabetic retinopathy: disruption of ocular physiology and visual functions by inflammatory gene products and pyroptosis. Front Physiol 2018; 9: 1268.

20. Nita M, Grzybowski A. The role of the reactive oxygen species and oxidative stress in the pathomechanism of the age-related ocular diseases and other pathologies of the anterior and posterior eye segments in adults. Oxid Med Cell Longev 2016; 2016: 3164734.

21. Matsushima S, Kuroda J, Ago T, Zhai P, Park JY, Xie LH, et al. Increased oxidative stress in the nucleus caused by Nox4 mediates oxidation of HDAC4 and cardiac hypertrophy. Circ Res 2013; 112(4): 651-63.

22. Chen J, Hui ST, Couto FM, Mungrue IN, Davis DB, Attie AD, et al. Thioredoxin-interacting protein deficiency induces Akt/Bcl-xL signaling and pancreatic beta-cell mass and protects against diabetes. FASEB J 2008; 22(10): 3581-94.

23. Lavieri R, Rubartelli A, Carta S. Redox stress unbalances the inflammatory cytokine network: role in autoinflammatory patients and healthy subjects. J Leukoc Biol 2016; 99(1): 79-86.

24. Saresella M, La Rosa F, Piancone F, Zoppis M, Marventano I, Calabrese E, et al. The NLRP3 and NLRP1 inflammasomes are activated in Alzheimer's disease. Mol Neurodegeneration 2016; 11: 23.

25. Mohamed IN, Ishrat T, Fagan SC, El-Remessy AB. Role of inflammasome activation in the pathophysiology of vascular diseases of the neurovascular unit. Antioxid Redox Signal 2015; 22(13): 1188-206. 